\title{
GERD prevalence in migraine patients and the implication for acute migraine treatment
}

\author{
Božena J. Katić · Wendy Golden · Roger K. Cady • \\ X. Henry Hu
}

Received: 23 July 2008/ Accepted: 27 October 2008/Published online: 14 November 2008

(C) Springer-Verlag 2008

\begin{abstract}
The objective of this study is to estimate the prevalence of gastroesophageal reflux disease (GERD) and heartburn in migraine patients and examine their use of non-steroidal anti-inflammatory drugs (NSAIDs) or aspirin-containing medications when treating acute migraine attacks. Responses from a web-based survey of migraine patients were matched to the same patient's responses on a general health survey. A total of 1,832 migraineurs (92.0\%) were successfully matched. A total of 403 migraineurs (22.0\%) reported having diagnosed GERD, 212 (11.6\%) reported diagnosed heartburn, and 290 (15.8\%) reported reflux symptoms but were undiagnosed. The most common prescription drugs used to treat migraines were triptans. First-line NSAID/aspirin medication use was $10.0 \%$ among diagnosed GERD and heartburn patients, $17.8 \%$ among undiagnosed patients, and $11.8 \%$ among GERD/heartburnfree migraineurs. In conclusion, almost half of migraineurs reported physician-diagnosed GERD and heartburn or symptoms of these conditions. Use of NSAID medications for migraine is fairly common among diagnosed GERD patients and more so for those with undiagnosed GERD
\end{abstract}

\section{B. J. Katić}

Center for Pharmaceutical Health Services Research,

Temple University School of Pharmacy, Philadelphia, PA, USA

W. Golden - X. H. Hu

Global Outcomes Research, Merck \& Co., Inc.,

Whitehouse Station, NJ, USA

R. K. Cady

Headache Care Center, Springfield, MO, USA

B. J. Katić $(\bowtie)$

One Merck Drive, Mailstop WS-2E65,

Whitehouse Station, NJ 08889, USA

e-mail: bkatic@temple.edu; bozena_katic@merck.com symptoms. Physicians should minimize prescribing NSAIDs or NSAID-containing acute migraine medications in this population.

Keywords Comorbidity - GERD - Migraine treatment . NSAID $\cdot$ Heartburn $\cdot$ Coprevalence

\section{Introduction}

Migraine, which currently affects 17 and $5.6 \%$ of women and men, respectively, in the US, is highly prevalent within the general population [1]. While migraine headache has a substantial impact on both the individual and society, it remains under-diagnosed and under-treated [1-3]. Accurate assessment of the clinical presentation of migraine and its co-prevalent conditions can help to guide physicians to maximize the therapeutic benefit of migraine treatment while minimizing untoward side effects.

Prior research has documented associations between migraine and a number of other conditions at greater prevalence than might be expected. Common comorbidities of migraine include anxiety, depression, epilepsy, insomnia, obesity, cardiovascular disease and stroke [4-7]. Migraine has also been linked to several gastrointestinal disorders including colitis, irritable bowel syndrome and peptic ulcer disease [8-10]. Research examining the coprevalence of headaches and common gastrointestinal disorders such as gastroesophageal reflux disease (GERD) and heartburn is scarce, although available studies have supported an increased prevalence of these conditions among migraineurs $[10,11]$. While the comorbidity of GERD and migraine is not known, a population-based study found that GERD affects between 13.2 and $20 \%$ of the US population [12]. 
The presence of gastroesophageal reflux disorder among migraineurs may present a unique treatment challenge for clinicians as several commonly used acute migraine medications, including non-steroidal anti-inflammatory drugs (NSAIDs) and aspirin, are known to cause gastrointestinal toxicity and exacerbation of erosive esophageal conditions [13]. NSAIDs and aspirin-containing medications (collectively referred to as NSAIDs) are commonly used by patients as either stand-alone acute migraine treatment or as part of a combination regimen (co-administered with another anti-migraine medication or as a rescue medication when first-line medication fails) [14]. Furthermore, prescription of NSAID use has been found to nearly double the risk of developing GERD in a controlled analysis of Medicaid users [15]. Undetected GERD among migraineurs may lead to the injudicious use of NSAIDs for headache and other pain, which may aggravate their underlying condition and increase healthcare resource utilization on the part of this population $[13,16]$. This study aims to assess the one-time prevalence of self-reported diagnosed GERD and heartburn symptoms from a population-based sample of migraineurs, and to examine acute migraine medication use by migraine patients with these conditions, particularly the prescription and over-thecounter (OTC) use of NSAIDs and aspirin.

\section{Methods}

Study population and data collection

A cross-sectional, web-based migraine survey was administered between November 2006 and January 2007. Participants must have previously reported physiciandiagnosed migraine on the 2006 National Health and Wellness Survey (NHWS), a nationwide survey of consumer healthcare attitudes, opinions, and self-reported diseases and conditions. The NHWS employs quota sampling based on age and race, and invitations are sent to the representative men and women within each quota for a total sample of 7,000 respondents per month. Inclusionary criteria were United States residency, 18 years of age or older, the ability to read and write English, agreement (consent) to study participation and not having participated in the survey during a previous month in 2006. Participants were excluded if any of the above criteria was not met.

After consenting, study participants were instructed to complete the migraine survey within $24 \mathrm{~h}$ of resolution of their next migraine attack, if such an attack occurred within 7 days of receipt of the study invitation. Migraine survey questions solicited demographic information, migraine history and treatment, presence of migraine-associated symptoms and current headache frequency and severity from participants. Information on GERD and heartburn conditions was retrieved by matching individual participant responses to the full NHWS dataset through unique identification numbers. The survey received central IRB approval (Essex IRB, Lebanon, NJ) and was fully compliant with all applicable state and federal privacy laws as well as the Health Insurance Portability and Accountability Act (HIPAA).

\section{Measures}

\section{GERD and heartburn diagnosis}

The prevalence of GERD or heartburn was ascertained by the NHWS question "Which of the following conditions have you experienced in the past 12 months?" This was followed by a checklist of diseases and conditions including GERD and, separately, heartburn. If GERD was reported, the follow-up question "Has your GERD been diagnosed by a physician?" was asked. Similarly, if heartburn was reported, participants were asked "Has your heartburn ever been diagnosed by a physician?" Patients who reported being diagnosed with both GERD and heartburn were classified as having diagnosed GERD. Migraineurs were classified into four mutually exclusive sub-populations: (1) those with physician-diagnosed GERD, (2) physician-diagnosed heartburn only (but not GERD), (3) undiagnosed symptoms of GERD or heartburn (symptomatic GERD/heartburn) and (4) migraineurs without GERD or heartburn symptoms (i.e., GERD/heartburn-free).

\section{Medication use}

Acute migraine medication use for participants' most recent attack was ascertained by the question "What first migraine medication did you take?" Responses were classified into one of eight categories: triptans, prescription NSAIDs or NSAID-containing combinations, prescription aspirin-containing combinations, opioids and opioid-containing combinations, other prescription medications, OTC NSAIDs, OTC aspirin and aspirin-containing combinations and other OTC medications. For the purposes of this study, a combination medication containing both an opioid and an NSAID or an opioid and aspirin were classified as an NSAID-containing combination and an aspirin-containing combination, respectively.

\section{Data analysis}

A secondary data analysis of the matched dataset was conducted. Demographic and migraine characteristics for each of the migraine sub-populations were compared using 
Chi-square statistics for categorical variables, and where appropriate, Fisher's exact tests. For categorical variables, individual $P$ values were adjusted in a step-up fashion using Benjamin-Hochberg's method to correct for multiple comparisons [17]. Continuous variables were analyzed by comparing adjusted group means using analysis of variance (ANOVA) for non-normal distributions. Post-hoc analyses for differences between group means were identified with Scheffe's multiple comparisons procedure. $P$ values less than or equal to 0.05 were considered statistically significant, and only $P$ values less than 0.10 were reported in results. Percentages were rounded to the nearest tenth when presented. All analyses were generated with SAS Statistical Analysis Software, Version 9.1

\section{Results}

A total of 4,000 potential eligible migraineurs were contacted. Of them, 2001 completed the online migraine attack survey (50.0\%). A total of 1,832 migraineurs (92.0\%) were matched to their responses on the 2006 NHWS survey, thus providing data on potential health-related comorbidities. The resulting classification of the four mutually exclusive migraine sub-populations derived from the total sample is presented in Fig. 1.

Of 1,832 matched migraine patients (mean age 41.1 years; $73 \%$ female), 403 (22.0\%) reported physiciandiagnosed GERD, 212 (11.6\%) reported physician-diagnosed heartburn, and an additional 290 (15.8\%) reported having symptoms of GERD and/or heartburn but were not diagnosed. Of the 403 respondents diagnosed with GERD, $78 \%(n=291)$ reported having heartburn symptoms, and the majority $(68 \%, n=274)$ also reported being diagnosed with heartburn. In total, 905 (49.4\%) reported either diagnosed or symptomatic GERD and/or heartburn (Fig. 1).

Sample characteristics of the study participants are presented in Table 1. Several differences were observed among the migraine sub-populations. Migraineurs with physician-diagnosed GERD were older $(P=0.05)$ and more likely male $(P=0.02)$ than either migraineurs with undiagnosed GERD or heartburn symptoms or those who were GERD/heartburn free. Those with diagnosed GERD were also more likely to suffer from severe migraines $(P=0.03)$ and chronic daily headaches $(P<0.01)$ than either migraineurs with undiagnosed symptoms of GERD/ heartburn or those without GERD/heartburn. Migraineurs with diagnosed heartburn reported a greater frequency of migraine attacks per month $(P=0.05)$, more severe migraines $(P=0.03)$, and having tension or chronic daily headaches $(P=0.04$ and $<0.01$, respectively) more often than either migraineurs with undiagnosed symptoms of
GERD/heartburn or those who were GERD/heartburn-free. Migraineurs with diagnosed GERD were somewhat more likely to have nausea at headache onset than the rest of the sample $(P=0.06)$. Patients with undiagnosed GERD/ heartburn symptoms were significantly less likely to be under the care of a neurologist or headache specialist for their migraines compared to those with diagnosed GERD, diagnosed heartburn, or GERD/heartburn-free individuals $(P<0.01)$. They were also marginally less likely to have health insurance which covers prescription medications when compared to either the diagnosed or disease-free populations, but this finding was not significant $(P=0.08)$.

Upon further examination of GERD in migraineurs with other primary headaches, it was found that GERD diagnosis was more prevalent among patients with sinus headache or tension-type headache. Of the 1,832 study participants, $802(43.7 \%)$ reported also having sinus headache.

In total, $27.2 \%(n=218)$ of those with sinus headache reported GERD diagnosis compared to $20.1 \%$ of those without sinus headache $(n=143 ; P=0.005)$. Similarly, 936 migraineurs $(51.1 \%)$ had co-prevalent tension-type headache. Of them, 26.5\% $(n=248)$ reported being diagnosed with GERD in contrast to $19.5 \%$ of migraineurs without tension-type headache $(n=113 ; P=0.005)$.

Distribution of first migraine medication used by the $1,779(97.1 \%)$ respondents who reported taking any medication for their last migraine attack is shown in Table 2. The majority of migraineurs from each sub-population used a triptan as their first migraine medication $(68.9 \%$ of total). Migraineurs with diagnosed heartburn or diagnosed GERD reported significantly more opioid and other prescription medication use $(P<0.01)$ compared to the rest of the sample, while symptomatic but undiagnosed GERD/ heartburn migraineurs took significantly more aspirincontaining drugs than did the diagnosed groups $(P<0.01)$. This relationship remained significant after adjustment for all pair-wise comparisons.

OTC NSAIDs and aspirin-containing medications were used more commonly than their prescription counterparts across all migraine sub-populations (Table 2). There was $1.5 \times$ more frequent use of OTC versus prescription aspirin-containing preparations among those with diagnosed GERD (4.1 vs. $2.8 \%$ ). OTC aspirin-containing use was $5.2 \times$ more common than prescription product use among those with undiagnosed symptoms of GERD or heartburn (11.0 vs. $2.1 \%$ ). The OTC to prescription ratio for NSAID use ranged from 1.6 to 2.1 greater OTC use across the four migraine sub-populations.

Upon combining prescription and OTC preparations of NSAIDs (and aspirins), significantly more migraineurs with undiagnosed symptoms of GERD or heartburn $(17.8 \%)$ chose an NSAID/aspirin product as their first-line 


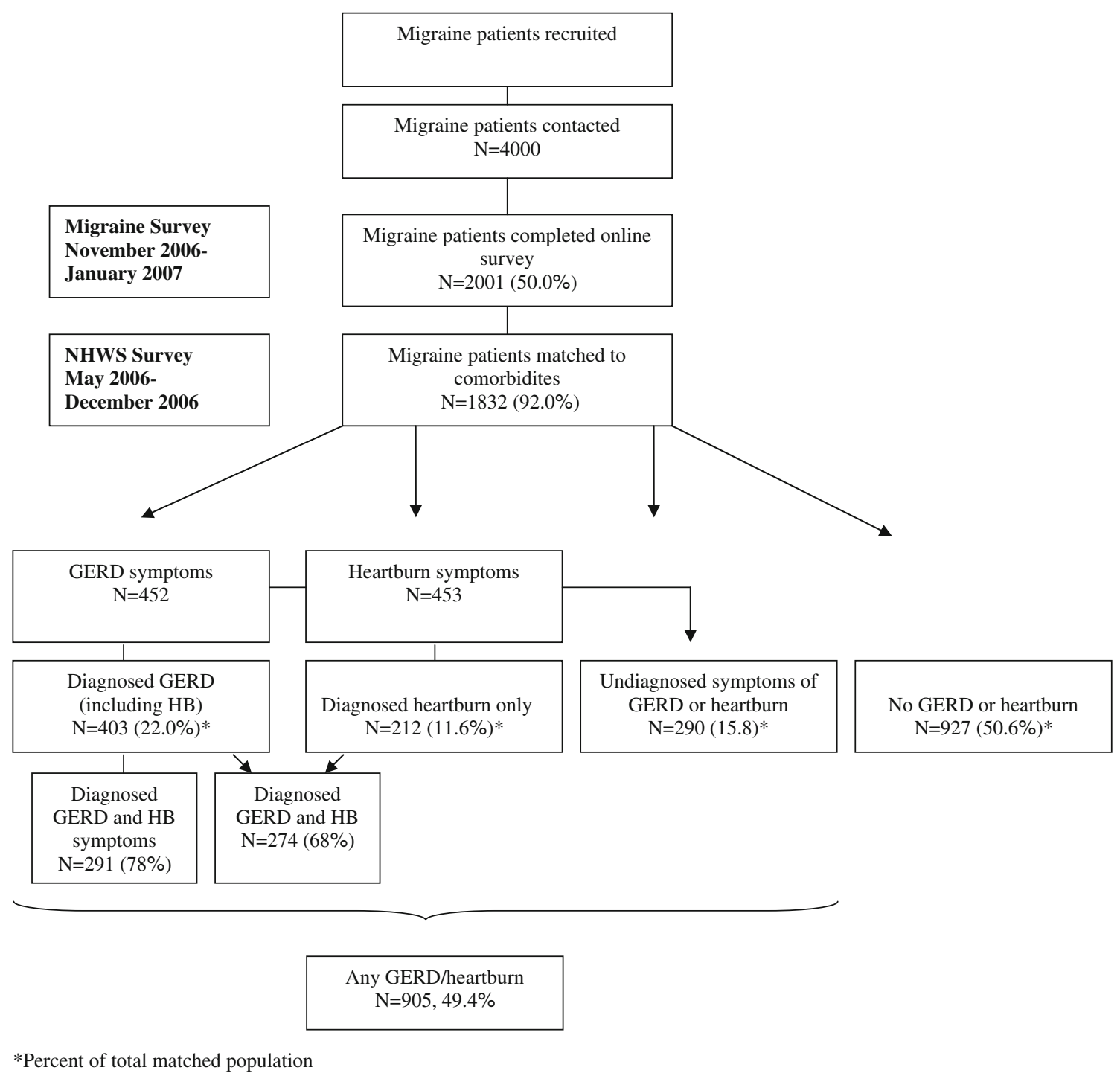

Fig. 1 Migraine sample

migraine medication compared to those with physiciandiagnosed GERD (10.0\%), physician-diagnosed heartburn $(10.1 \%)$, or those who were GERD/heartburn free $(11.8 \%$; $P<0.01)$.

\section{Discussion}

In this cross-sectional study, the self-reported prevalence of physician-diagnosed GERD among migraineurs was $22.0 \%$, and the prevalence of diagnosed heartburn was $11.6 \%$. Nearly half $(49.4 \%)$ of the total migraine sample reported being diagnosed with GERD or heartburn or experiencing symptoms of these conditions within the past year. The rates found in this study appear similar to estimates within the general US population, which approximate $20 \%$ for GERD [18] and up to $44 \%$ for occasional heartburn symptoms [19]. Migraineurs with comorbid tension-type or sinus headache were significantly more likely to report being diagnosed with GERD than migraineurs who did not report having additional headaches, a finding cited by previous research $[10,20]$.

An increased prevalence of gastroesophageal reflux among migraine sufferers is supported by a report by Featherstone [11] and is similar to the more recent findings reported by Aamodt and colleagues [10], who found a $42.6 \%$ prevalence of reflux symptoms among Norwegian migraineurs, an association which strengthened as the amount of reflux symptoms increased. A small observational study in Italy [21] aimed to discover the rationale for this apparent association, and found that migraineurs with functional dyspepsia could be characterized by their hypersensitivity to gastric distention and a lower discomfort threshold after meals when compared to both 
Table 1 Sample characteristics of study participants, by migraine sub-populations ( $n=1,832)$

\begin{tabular}{|c|c|c|c|c|c|}
\hline & $\begin{array}{l}\text { Diagnosed GERD } \\
n=403\end{array}$ & $\begin{array}{l}\text { Diagnosed } \\
\text { heartburn } n=212\end{array}$ & $\begin{array}{l}\text { Symptoms of GERD or } \\
\text { heartburn } n=290\end{array}$ & $\begin{array}{l}\text { No GERD or heartburn } \\
\text { symptoms } n=927\end{array}$ & $P$ value \\
\hline \multicolumn{6}{|l|}{ Demographic characteristics } \\
\hline Age, mean (SD) & $43.9(11.1)^{\mathrm{c}}$ & $42.1(11.8)$ & $39.2(11.5)$ & $40.3(11.8)$ & 0.05 \\
\hline Female $(\%)$ & $69.5^{\mathrm{c}}$ & $67.0^{\mathrm{c}}$ & 75.9 & 75.1 & 0.02 \\
\hline College graduate $(\%)$ & 38.9 & 41.5 & 45.2 & 46.4 & \\
\hline $\begin{array}{l}\text { Prescription drug insurance } \\
(\%)\end{array}$ & 88.6 & 86.3 & 83.8 & 88.8 & 0.08 \\
\hline \multicolumn{6}{|l|}{ Migraine characteristics } \\
\hline Age at diagnosis, mean (SD) & $28.3(12.1)$ & $26.7(11.5)$ & $27.2(10.9)$ & $26.7(10.6)$ & \\
\hline \multicolumn{6}{|l|}{ General migraine severity (\%) } \\
\hline Mild & 1.7 & 1.4 & 2.8 & 3.5 & \\
\hline Moderate & 37.7 & 39.2 & 47.2 & 50.0 & \\
\hline Severe & $60.5^{\mathrm{c}}$ & $59.4^{\mathrm{c}}$ & 50.0 & 53.4 & 0.03 \\
\hline $\begin{array}{l}\text { Attack frequency per } \\
\text { month }^{\mathrm{a}} \text {, mean (SD) }\end{array}$ & $6.2(6.3)$ & $6.7(6.7)^{\mathrm{b}}$ & $5.1(5.5)$ & $5.7(6.1)$ & 0.05 \\
\hline \multicolumn{6}{|l|}{ Other primary headaches $(\%)$} \\
\hline Tension & 80.9 & $84.4^{\mathrm{b}}$ & 79.7 & 76.5 & 0.04 \\
\hline Cluster & 36.5 & 40.6 & 37.2 & 35.0 & \\
\hline Chronic daily & $40.0^{\mathrm{c}}$ & $39.6^{\mathrm{c}}$ & 31.0 & 30.1 & 0.001 \\
\hline Sinus & 69.2 & 68.9 & 64.8 & 64.7 & \\
\hline \multicolumn{6}{|c|}{ Symptoms at headache onset $(\%)$} \\
\hline Nausea & 63.0 & 58.5 & 60.3 & 55.5 & 0.06 \\
\hline Vomiting & 13.2 & 10.7 & 11.0 & 12.2 & \\
\hline \multicolumn{6}{|l|}{ Migraine provider type (\%) } \\
\hline $\begin{array}{l}\text { Neurologist/headache } \\
\text { specialist }\end{array}$ & 25.6 & 22.2 & $16.5^{\mathrm{c}}$ & 24.2 & $<0.01$ \\
\hline
\end{tabular}

a Non-normal distribution

b Differences between groups 2 and 3 or groups 2 and 4

${ }^{c}$ Differences between groups 1 and 3, 1and 4; and/or groups 2 and 3, 2 and 4

dyspeptics without migraine and healthy volunteers. Furthermore, clinical case studies have concluded that gastric reflux disorders can trigger migraine headache originating from specific locations [22].

The mechanism by which GERD and reflux symptoms are increased among migraineurs is not clear, but is plausibly related to the common etiology of both disorders. Autonomic nervous system (ANS) dysfunction has been shown to play a role in the pathogenesis of both reflux disorders and migraine attacks [23, 24], which may explain the overlap in GERD and migraine symptomatology. Pathophysiologically, abnormal visceral mechanosensory and vagal function has been found to play a role in dyspeptic patients, and similarly, disturbances in visceral nerves have been implicated in migraine [25, 26]. Neuropeptides such as the calcitonin gene-related peptide (CGRP) have also played an important role in this association. CGRP is a neurotransmitter which helps to regulate receptive relaxations in the stomach and gastrointestinal tract, and studies find it is increased during migraine attack [27]. It has been hypothesized that disorders of the reservoir functions mediated by CGRP may result in symptoms of functional dyspepsia [28].

This study also observed that migraineurs with diagnosed GERD or heartburn conditions reported a slightly higher incidence of nausea than their counterparts without these conditions, an association which has been documented by the literature $[10,11]$. In a clinic-based sample of Italian patients referred for endoscopy, increased prevalence of migraine was reported in dysmotility-like (e.g., gastroparesis), but not reflux-like, dyspeptic patients [29]. Nonetheless, other studies have found that delayed gastric emptying or gastroparesis itself plays a significant role in the evolution of GERD [30]. It is estimated that gastroparesis is present in 15\% of GERD patients, although underdiagnosed. Research has confirmed that gastric statis is present among migraineurs both during and outside of migraine attacks [31]; which is suggestive of underlying 
Table 2 Distribution of first medication used, by migraine sub-populations $(n=1,779)$

\begin{tabular}{|c|c|c|c|c|c|}
\hline Medication type (\%) & $\begin{array}{l}\text { Diagnosed } \\
\text { GERD } \\
n=403\end{array}$ & $\begin{array}{l}\text { Diagnosed } \\
\text { heartburn } \\
n=212\end{array}$ & $\begin{array}{l}\text { Symptoms of } \\
\text { GERD/heartburn } \\
n=290\end{array}$ & $\begin{array}{l}\text { No GERD or } \\
\text { heartburn symptoms } \\
n=927\end{array}$ & $P$ value \\
\hline Triptans & 66.7 & 66.2 & 66.9 & 71.1 & \\
\hline NSAID and NSAID-containing & 3.1 & 4.8 & 4.6 & 5.6 & \\
\hline Prescription & 1.0 & 1.5 & 1.8 & 1.8 & \\
\hline OTC & 2.1 & 3.4 & 2.9 & 3.8 & \\
\hline Aspirin and aspirin-containing & 6.9 & 5.3 & $13.2^{\mathrm{a}}$ & 6.2 & $<0.01$ \\
\hline Prescription & 2.8 & 1.5 & 2.1 & 1.1 & \\
\hline OTC & 4.1 & 3.9 & 11.0 & 5.1 & \\
\hline $\begin{array}{l}\text { Total NSAID and aspirin-containing } \\
\text { medication }\end{array}$ & 10.0 & 10.1 & $17.8^{\mathrm{a}}$ & 11.8 & 0.001 \\
\hline Opioids & $6.2^{\mathrm{c}}$ & $7.7^{\mathrm{b}}$ & 2.1 & 4.4 & $<0.01$ \\
\hline Prescription (other) & $15.0^{\mathrm{c}}$ & $15.0^{\mathrm{b}}$ & 11.0 & 10.8 & $<0.01$ \\
\hline OTC (other) & 2.1 & 1.0 & 2.1 & 1.8 & \\
\hline
\end{tabular}

${ }^{a}$ Differences in group 3

b Differences in group 2

${ }^{c}$ Differences in group 1

gastroesophageal disorder as much as it is of migraine. A recent population-based study found that both nausea and reflux can occur concurrently among headache sufferers with high symptom loads, despite the lack of common etiology between the two symptoms [10]. Given the substantial overlap between dysmotility-like and reflux-like dyspepsia among those who experience any dyspepsic symptoms [32], we believe the findings of our study support, at the very least, an association between dyspepsic symptoms and migraine among those who may suffer from pre-existing gastroesophageal conditions.

Upon examining migraine medication used at last attack, we found a relatively high frequency of NSAID or opioid use among migraineurs, classes of drugs that are both associated with upper as well as lower GI symptoms. Approximately $10 \%$ of migraineurs with physician-diagnosed GERD and $17 \%$ of migraineurs with undiagnosed GERD or heartburn symptoms reported taking an NSAID, aspirin, or an aspirin-containing product as their first-line abortive medication for their most recent migraine attack, and the rate of collective NSAID use among migraineurs with physician-diagnosed GERD was comparable to that of migraineurs without GERD or heartburn symptoms. Upper GI symptoms including heartburn, reflux and dyspepsia are common complaints in both prescription and OTC NSAID users $[16,33]$. NSAIDs have been shown to approximately double the risk of development of GERD [15, 34] and have also been shown to have other toxic effects on the esophagus. Case-control studies have found an association between NSAID consumption and esophageal strictures, and NSAIDs have been reported to cause pill-induced esophagitis, especially when the drugs are absorbed without water as they might be during a migraine attack [35]. As it is well-known, NSAIDs also increase the risk of gastroduodenal ulceration, bleeding, and perforation, even with OTC or low-dose formulations [36]. Given the high healthcare resource utilization associated with NSAID/ aspirin complications, the relatively frequent use of NSAIDs as acute therapy among migraineurs with physician-diagnosed GERD or heartburn symptoms generates some cause for clinical concern. In this study, we found that triptans were most commonly used; which, unlike NSAIDs, are drugs specifically indicated for migraine treatment. Triptans, on the other hand, are contraindicated in patients with ischemic heart disease or uncontrolled hypertension. Although triptans are generally considered safe when used appropriately according to the approved label [37], physicians should obtain completed medical histories in addition to migraine symptoms and carefully evaluate the benefit risk trade-off before prescribing either triptans or NSAIDs.

This study had several limitations which deserve mention. Primarily, the prevalence of physician-diagnosed GERD, heartburn symptoms, migraine and other headaches were made on the basis of a patient self-reporting diagnoses received by their doctor. However, we have no reason to suspect that migraineurs reported being diagnosed with GERD if they were not. Patient-reported data of this kind has been found to be reliable, and for the most part consistent, with physician reports [38, 39].

Due to the lack of control for potential confounding in this study, predictors linked to both GERD and migraine- 
such as anxiety, depression, smoking, alcohol intake, analgesic use or obesity-cannot be ruled out as an explanation for the rate of GERD/heartburn observed among this sample of migraineurs. But confounding seems unlikely to account for the entire association, as the positive relationship observed between migraine and reflux symptoms was only mildly attenuated after adjustment for anxiety, depression and chronic medication use previously [10]. Furthermore, another study which controlled for the effects of smoking, gender, alcohol use and analgesic intake in this association found no indication that the higher prevalence of dyspepsia among migraineurs was induced or modified by analgesic intake [26].

Given the cross-sectional nature this study and the absence of a control group, the secondary data analysis employed here could not establish a causal relationship between migraine and GERD/heartburn in either direction. We also recognize that the online survey format may have biased our sample of migraineurs against those who are older, of a lower SES level, or who do not have regular internet access.

Because medication data was only available for the last migraine attack experienced in this cross-sectional sample, we were unable to show the effects of chronic NSAID use by patients with GERD/heartburn; or the degree to which NSAIDs used for migraine headache either causally contribute to or worsen GERD over time. However, because only the first migraine medication taken following a migraine attack was recorded, our study likely significantly underestimates the cumulative use of NSAIDs, aspirin, and aspirin-containing medications during any given migraine attack. Ng-Mak and colleagues recently found that in addition to the $11 \%$ of migraineurs who treated their most recent attack exclusively with an NSAID, triptan/NSAID co-therapy and using an NSAID as rescue medication were common practices when both classes of medication were available to patients [14]. It was also shown that for the patients with migraine-associated nausea, triptan/NSAID therapy was seemingly less effective than triptan monotherapy in relieving nausea. Our study found a similar rate of first-line NSAID use for acute migraine therapy for both patients with physician diagnosed GERD or heartburn $(10.1 \%)$ and for those who were GERD/heartburn-free $(11.8 \%)$.

In addition to the apparent co-prevalence GERD or heartburn among migraineurs and their fairly frequent use of NSAIDs for migraine attacks, several other findings are apparent from these results. We found that the most commonly used NSAID for the acute treatment of migraine was an aspirin or aspirin-containing product. The broad availability of OTC aspirin products makes them popular treatment choices for migraine as well as tension and other headache types. Low-dose aspirin is also regularly used among the general population for the prevention of stroke and cardio-vascular events [40]. However, it is unlikely that we included preventative aspirin use in our medication type analysis, as aspirins taken for this purpose are generally taken at too low a dosage strength to be used as a first-line acute migraine treatment.

While the majority of our sample population was diagnosed with GERD $(n=403)$ as opposed to diagnosed with heartburn $(n=212)$, diagnosed heartburn accounted for part of the significant differences seen with regard to migraine characteristics and medication use among the diagnosed population. Diagnosed heartburn was associated with greater migraine severity, increased the presence of both tension and chronic daily headaches and slightly more opioid medication use among the combined diagnosed population. While the absolute overlap of these highly similar disorders is difficult to accurately assess, we found that $72 \%$ of the diagnosed GERD population reported to have heartburn symptoms, and $68 \%$ reported being codiagnosed with heartburn. Given the high rate of overlap between symptomatic or 'diagnosed' GERD and heartburn, the true rate of GERD disorder is likely underestimated in this migraine sample.

A substantial proportion of those who reported undiagnosed GERD or heartburn symptoms used OTC NSAID or aspirin regimens for first-line treatment of their migraine attack; moreover, NSAID use was apparent in this group than in the GERD or heartburn-free population. The undiagnosed population was also significantly less likely to see a headache specialist for their migraine symptoms than were any of the other groups, and also marginally less likely to have prescription drug insurance. We would expect that these structural barriers increase their reliance on OTC aspirin products. However, reliance on OTC products may also be partially explained by the undiagnosed patient's perception of his/her own illness severity and their underlying belief that OTC medications are effective in treating existing symptoms. Studies have found that a high proportion of individuals with GERD perceive their symptoms to be due to a trivial condition, and recently research has found that a large majority (close to 78\%) of patients with undiagnosed GERD symptoms relied on OTC medications to alleviate their symptoms [41]. This strong reliance on OTC medications may make undiagnosed GERD sufferers less likely to see a healthcare provider and obtain a diagnosis for their symptoms to begin with.

While NSAIDs can be efficacious as acute migraine therapy, medications containing this class of drugs may not be appropriate for all patients. The common finding of GERD among migraineurs coupled with their relatively frequent use of NSAIDs should encourage physicians who treat migraine to specifically inquire about the presence of upper GI symptoms in patients. Approximately two-thirds 
of the NSAID, aspirin and aspirin-containing products reported in this study were OTC, so physicians may be unaware of the use of these medications during a migraine attack; particularly, since the majority of OTC medication use, unlike triptan or other prescription analgesic use, goes unreported. Some GI patients medicate with OTC NSAIDs for pain, and take additional OTC GI medications for GI side effects [16] which may increase their risk of drugrelated interactions, unknown to the clinician. Because migraineurs may vary their medication regimen from one attack to the next [42], the need for increased physicianpatient communication regarding medication usage and the risks and benefits of various migraine and heartburn-alleviating therapies may be critical for successsful migraine management. Discussing the functional disability and symptoms associated with migraine during initial consultation has also been found to have a powerful impact on physicians' perception of illness severity as well as on their choice of subsequent treatments for the patient [43].

To our knowledge, this is the first observational study that estimates the co-prevalence of suspected GERD or heartburn among migraine patients and documents their use of anti-migraine medication during acute migraine attack. The presence of GERD and heartburn symptoms among migraineurs, coupled with their relatively frequent use of NSAIDs, should encourage physicians to inquire about the use of OTC analgesics among this population and use discretion prior to prescribing appropriate treatment.

Acknowledgments This research study was funded by Merck \& Co., Inc.

Conflict of interest B. J. Katic is a Temple University research fellow sponsored by Merck. W. Golden and X. Henry Hu are both employed by Merck \& Co., Inc. Roger K. Cady is a consultant/ advisor for Merck and is involved in research studies for which his academic institution has received research grants. He has no stock or equity in any pharmaceutical company.

\section{References}

1. Diamond S, Bigal ME, Silberstein S, Loder E, Reed M, Lipton RB (2006) Patterns of diagnosis and acute and preventive treatment for migraine in the United States: results from the American Migraine Prevalence and Prevention study. Headache J Head Face Pain 47(3):355-363

2. Cady RK (1999) Diagnosis and treatment of migraine. Clin Cornerstone 1(6):21-32

3. Bigal ME, Lipton RB, Stewart WF (2004) The epidemiology and impact of migraine. Curr Neurol Neurosci Rep 4(2):98-104

4. Bigal ME, Lipton RB, Holland PR, Goadsby PJ (2007) Obesity, migraine, and chronic migraine: possible mechanisms of interaction. Neurology 68(21):1851-1861

5. Kurth T (2007) Associations between migraine and cardiovascular disease. Expert Rev Neurother 7(9):1097-1104
6. Sevillano-Garcia MD, Manso-Calderon R, Cacabelos-Perez P (2007) Comorbidity in the migraine: depression, anxiety, stress and insomnia. Rev Neurol 45(7):400-405

7. Merikangas KR, Fenton BT, Cheng SH, Stolar MJ, Risch N (1997) Association between migraine and stroke in a large-scale epidemiological study of the United States. Arch Neurol 54(4):362-368

8. Jones R, Lydeard S (1992) Irritable bowel syndrome in the general population. BMJ 304(6819):87-90

9. Merikangas KR, Fenton BT (1994) Comorbidity of migraine with somatic disorders in a large-scale epidemiologic study in the United States (47):301-314

10. Aamodt AH, Stovner LJ, Hagen K, Zwart JA (2008) Comorbidity of headache and gastrointestinal complaints. The Head-HUNT Study. Cephalalgia 28(2):144-151

11. Featherstone HJ (1985) Medical diagnoses and problems in individuals with recurrent idiopathic headaches. Headache 25(3): 136-140

12. Locke GR III, Talley NJ, Fett SL, Zinsmeister AR, Melton LJ III (1997) Prevalence and clinical spectrum of gastroesophageal reflux: a population-based study in Olmsted County, Minnesota. Gastroenterology 112(5):1448-1456

13. Gutthann SP, Guez LA, Raiford DS (1997) Individual nonsteroidal antiinflammatory drugs and other risk factors for upper gastrointestinal bleeding and perforation. Epidemiology 8(1):1824

14. Ng-Mak DS, Hu XH, Chen YT, Ma L (2008) Acute migraine treatment with oral triptans and NSAIDS in a managed care population. Headache 48(8):1176-1185

15. Kotzan J, Wade W, Yu HH (2001) Assessing NSAID prescription use as a predisposing factor for gastroesophageal reflux disease in a Medicaid population. Pharm Res 18(9):1367-1372

16. Thomas J, Straus WL, Bloom BS (2002) Over-the-counter nonsteroidal anti-inflammatory drugs and risk of gastrointestinal symptoms. Am J Gastroenterol 97(9):2215-2219

17. Hsu JC (2007) Hochberg's step-up method: cutting corners off Holm's step-down method. Biometrika 94(4):965-975

18. Dent J, El Serag HB, Wallander MA, Johansson S (2005) Epidemiology of gastro-oesophageal reflux disease: a systematic review. Gut J Br Soc Gastroenterol 54(5):710-717

19. Shaheen N, Provenzale D (2003) The epidemiology of gastroesophageal reflux disease. Am J Med Sci 326(5):264-273

20. DiBaise JK, Olusola BF, Huerter JV, Quigley EM (2002) Role of GERD in chronic resistant sinusitis: a prospective, open label, pilot trial. Am J Gastroenterol 97(4):843-850

21. Pucci E, Di Stefano M, Miceli E, Corazza GR, Sandrini G, Nappi G (2005) Patients with headache and functional dyspepsia present meal-induced hypersensitivity of the stomach. J Headache Pain 6(4):223-226

22. Spierings EL (2002) Reflux-triggered migraine headache originating from the upper gum/teeth. Cephalalgia 22(7):555-556

23. Peroutka SJ (2004) Migraine: a chronic sympathetic nervous system disorder. Headache J Head Face Pain 44(1):53-64

24. Karamanolis G, Sifrim D (2007) Developments in pathogenesis and diagnosis of gastroesophageal reflux disease. Curr Opin Gastroenterol 23(4):428-433

25. Moskowitz MA (1990) Basic mechanisms in vascular headache. Neurol Clin 8(4):801-815

26. Kurth T, Holtmann G, Neufang-Hnber J, Gerken G, Diener HC (2006) Prevalence of unexplained upper abdominal symptoms in patients with migraine. Cephalalgia 26(5):506-510

27. Edvinsson L (2005) Clinical data on the CGRP antagonist BIBN4096BS for treatment of migraine attacks. Cns Drug Rev 11(1):69-76

28. Arakawa T, Uno H, Fukuda T, Higuchi K, Kobayashi K, Kuroki $\mathrm{T}$ (1997) New aspects of gastric adaptive relaxation, reflex after 
food intake for more food: involvement of capsaicin-sensitive sensory nerves and nitric oxide. J Smooth Muscle Res 33(3):8188

29. Meucci G, Radaelli F, Prada A, Bortoli A, Crotta S, Cerrato C, Minoli G (2005) Increased prevalence of migraine in patients with uninvestigated dyspepsia referred for open-access upper gastrointestinal endoscopy. Endoscopy 37(7):622-625

30. Horowitz M, Su YC, Rayner CK, Jones KL (2001) Gastroparesis: prevalence, clinical significance and treatment. Can J Gastroenterol 15(12):805-813

31. Aurora SK, Kori SH, Barrodale P, McDonald SA, Haseley D (2006) Gastric stasis in migraine: more than just a paroxysmal abnormality during a migraine attack. Headache J Head Face Pain 46(1):57-63

32. Talley NJ, Zinsmeister AR, Schleck CD, Melton LJ III (1992) Dyspepsia and dyspepsia subgroups: a population-based study. Gastroenterology 102(4 Pt 1):1259-1268

33. Laine L (2002) The gastrointestinal effects of nonselective NSAIDs and COX-2-selective inhibitors. Semin Arthritis Rheum 32(3):25-32

34. Voutilainen M, Sipponen P, Mecklin JP, Juhola M (2000) Gastroesophageal reflux disease: prevalence, clinical, endoscopic and histopathological findings in 1, 128 consecutive patients referred for endoscopy due to dyspeptic and reflux symptoms. Digestion 61(1):6-13

35. Bigard MA, Pelletier AL (2004) Esophageal complications of non steroidal antiinflammatory drugs. Gastroenterol Clin Biol $28: 358-361$
36. Kim SL, Hunter JG, Wo JM, Davis LP, Waring JP (1999) NSAIDs, aspirin, and esophageal strictures: are over-the-counter medications harmful to the esophagus? J Clin Gastroenterol 29(1):32-34

37. Dodick DW, Martin VT, Smith T, Silberstein S (2004) Cardiovascular tolerability and safety of triptans: a review of clinical data. Headache 44(s1):S20-S30

38. Mulleners WM, Aurora SK, Chronicle EP, Stewart R, Gopal S, Koehler PJ (2001) Self-reported photophobic symptoms in migraineurs and controls are reliable and predict diagnostic category accurately. Headache J Head Face Pain 41(1):31-39

39. Iversen L, Hannaford PC, Godden DJ, Price D (2007) Do people self-reporting information about chronic respiratory disease have corroborative evidence in their general practice medical records? A study of intermethod reliability. Prim Care Resp J 16(3):162168

40. Tseeng S, Arora R (2008) Aspirin resistance: biological and clinical implications. J Cardiovasc Pharmacol Ther 13(1):5-12

41. Ducrotte P, Liker HR (2007) How do people with gastrooesophageal reflux disease perceive their disease? Results of a multinational survey. Curr Med Res Opin 23(11):2857-2865

42. Malik SN, Hopkins M, Young WB, Silberstein SD (2006) Acute migraine treatment: patterns of use and satisfaction in a clinical population. Headache J Head Face Pain 46(5):773-780

43. Holmes WF, MacGregor EA, Sawyer JP, Lipton RB (2001) Information about migraine disability influences physicians' perceptions of illness severity and treatment needs. Headache $\mathbf{J}$ Head Face Pain 41(4):343-350 